\title{
ULTRAFILTER CONVERGENCE IN ORDERED TOPOLOGICAL SPACES
}

\author{
PAOLO LIPPARINI
}

\begin{abstract}
We characterize ultrafilter convergence and ultrafilter compactness in linearly ordered and generalized ordered topological spaces. In such spaces, and for every ultrafilter $D$, the notions of $D$-compactness and of $D$ pseudocompactness are equivalent. Any product of initially $\lambda$-compact generalized ordered topological spaces is still initially $\lambda$-compact. On the other hand, preservation under products of certain compactness properties is independent from the usual axioms for set theory.
\end{abstract}

Once upon a time people red papers and sometimes quoted them. Now people quote papers and sometimes read them.

\section{INTRODUCTION}

It is well-known that many covering properties, which in general are distinct, turn out to be equivalent for linearly ordered topological spaces (henceforth, LOTS, for short). For example, a LOTS is pseudocompact if and only if it is countably compact, if and only if it is sequentially compact. See Gulden, Fleischman and Weston [GFW70 and Purisch Pur73 for these and more general results, also involving uncountable cardinals and dealing with product theorems, as well. Most of the above results generalize to GO spaces, short for generalized ordered topological spaces; see [Pur73] and Lip13, or below.

Here we show that, for every ultrafilter $D, D$-compactness and $D$-pseudocompactness are equivalent in GO spaces (Remark 3.2, or Theorem 4.1(1) $\Leftrightarrow(5)$ ). The particular case when $D$ is an ultrafilter over $\omega$ is due to García-Ferreira and Sanchis GFS97, whose paper contains many other related theorems. More generally, we characterize $D$-converging sequences in GO spaces (Proposition 3.1(7)-(9)). We also show that the $D$-compactness of some GO space $X$ depends exclusively on the "decomposability spectrum" of $D$ and on the cardinal types of (pseudo-)gaps in $X$, equivalently, on the existence of non converging strictly monotone sequences of certain cardinal order types (Theorem 4.1).

Some of the results mentioned in the first paragraph can be obtained as corollaries of Theorem 4.1. For example, we get a proof that any product of initially $\lambda$-compact GO spaces is still initially $\lambda$-compact (Corollary 5.1). The result is quite surprising, since noncompact covering properties are usually not preserved by taking products. Just to state a significant example, the square of a Lindelöf LOTS is not necessarily

2010 Mathematics Subject Classification. 54F05, 54A20, 54B10, 54D20; 06A05, 54A35, $03 \mathrm{E} 75$.

Key words and phrases. Linearly ordered, generalized ordered topological space; ultrafilter convergence, compactness, pseudocompactness; (pseudo-)gap; converging $\nu$-sequence; (weak) $[\nu, \nu]$ compactness; (weak) initial $\lambda$-compactness; complete accumulation point; $\lambda$-boundedness; decomposable, descendingly complete, regular ultrafilter. 
Lindelöf. The Sorgenfrey line is an example which is a GO space. To get an example which is a LOTS, consider $\mathbb{R} \times \omega$ with the lexicographic order. Also, it is well-known that the product of two countably compact topological spaces is not necessarily countably compact, and many similar counterexamples are known for initial $\kappa$ compactness, when $\kappa$ is regular. See, e. g., Stephenson Ste84, Vaughan Vau84, Nyikos and Vaughan [NV87] and more references there. Hence, for $\kappa$ regular, preservation of initial $\kappa$-compactness under products is really a special property of GO spaces. In passing, let us mention that, on the other hand, when $\kappa$ is a singular strong limit cardinal, every product of initially $\kappa$-compact topological spaces is still initially $\kappa$-compact, by the celebrated Stephenson and Vaughan Theorem 1 in SV74.

In the particular case of LOTS, preservation of initial $\kappa$-compactness under products had originally been proved in GFW70, for every infinite cardinal $\kappa$. Not aware of Gulden, Fleischman and Weston's result, we originally have found a proof which holds for GO spaces, too, and which uses ultrafilter convergence Lip13. Later we realized that the theorem can also be proved by adapting some arguments from GFW70 (see the second proof of Corollary 5.1 here), hence the use of ultrafilters becomes unnecessary. However, luckily for scholars fond of ultrafilters, there are indeed compactness properties whose preservation under products (in GO spaces) involves ultrafilters in an essential way. For example, this is the case when considering simultaneously countable compactness and $[\lambda, \lambda]$-compactness. Considering preservation of such properties leads to statements independent from the usual axioms of set theory, statements which involve the existence of ultrafilters with an "unusual" descending completeness spectrum (equivalently, decomposability spectrum). See Corollary 6.1 for the general statement, and Corollary 6.4 for an explicit example.

Now a few words about the fate of [Lip13]. Since all the results proved there are subsumed by the present paper, we are not going to submit Lip13 elsewhere. However, we shall keep it available in archived form, since it might be useful for those looking for a direct proof of Corollaries 5.1 and 5.2 here, which essentially were the main theorems of [Lip13].

\section{Preliminaries}

We now recall the relevant definitions. A LOTS is a linearly ordered set endowed with the open interval topology. A $G O$ space is a linearly ordered set with a $T_{2}$ topology having a base of order-convex sets. GO spaces are exactly spaces which can be obtained as subspaces of LOTS; the notion is more general, since if $X$ is a LOTS, and $Y \subseteq X$, then the subset topology on $Y$ induced by the topology on $X$ might be finer than the order topology on $Y$ relative to the restriction of the order on $X$. See, e. g., BL02 for more informations and references about LOTS and GO spaces.

If $D$ is an ultrafilter over some set $I$, then a topological space $X$ is said to be $D$-compact if every $I$-indexed sequence $\left(x_{i}\right)_{i \in I}$ of elements of $X D$-converges to some $x \in X$, that is, $\left\{i \in I \mid x_{i} \in U\right\} \in D$, for every open neighborhood $U$ of $x$. The notion of ultrafilter convergence has proved particularly useful in the study of compactness properties of topological spaces, in particular with respect to preservation under products. Classical papers on the subject are Bernstein [Ber70, Ginsburg and Saks [GS75, and Saks [Sak78]. Excellent surveys of results proved 
until the mid '80's are Vaughan Vau84 and Stephenson Ste84. Further results can be found in Caicedo [Cai99] and Lip14, together with additional references.

Ginsburg and Saks GS75] made also a very effective use of the notion of a $D$ limit point of a sequence of subsets of a topological space, and introduced the notion of $D$-pseudocompactness. See also GF99]. If $\left(Y_{i}\right)_{i \in I}$ is a sequence of subsets of $X$, a point $x \in X$ is said to be a $D$-limit point of $\left(Y_{i}\right)_{i \in I}$ if $\left\{i \in I \mid U \cap Y_{i} \neq \emptyset\right\} \in D$, for every neighborhood $U$ of $x$ (thus when each $Y_{i}$ is a singleton we get back the notion of $D$-convergence). A topological space $X$ is $D$-pseudocompact if every sequence $\left(O_{i}\right)_{i \in I}$ of nonempty open sets of $X$ has a $D$-limit point. Clearly, we can equivalently relax the assumption that all the $O_{i}$ 's are nonempty to the assumption that $\left\{i \in I \mid O_{i} \neq \emptyset\right\} \in D$, since we can change those $O_{i}$ 's in a set not in $D$ without affecting $D$-limit points of the sequence (since $D$ is a filter).

Consider the following statement.

$\left(^{*}\right) A$ and $B$ are open subsets of a GO space $X, A \cup B=X$, and $a<b$, for every $a \in A$ and $b \in B$.

An ordered pair $(A, B)$ satisfying $\left(^{*}\right)$ is called a gap of $X$ in case that neither $A$ has a maximum, nor $B$ has a minimum. Here we are including end gaps in the definition of a gap, that is, we allow either $A$ or $B$ to be empty. An ordered pair $(A, B)$ satisfying $(*)$ is called a pseudo-gap in case that both $A$ and $B$ are nonempty, and either $A$ has a maximum and $B$ has no minimum, or $A$ has no maximum and $B$ has a minimum. Clearly pseudo-gaps can occur only in GO spaces which are not LOTS, since we are asking that $A$ and $B$ are open.

For a property $\mathcal{P}$, the expression " $X$ has no (pseudo-)gap satisfying $\mathcal{P}$ " shall be used as an abbreviation for " $X$ has neither a gap nor a pseudo-gap satisfying $\mathcal{P}$ ".

\section{D-Convergence of a Given SEQuence}

Proposition 3.1. Suppose that $X$ is a linearly ordered set, $D$ is an ultrafilter over some set $I$, and $\left(x_{i}\right)_{i \in I}$ is a sequence of elements of $X$. Set $A=\{x \in X \mid\{i \in$ $\left.\left.I \mid x<x_{i}\right\} \in D\right\}$, and $B=\left\{x \in X \mid\left\{i \in I \mid x_{i}<x\right\} \in D\right\}$. Then the following statements hold.

(1) $x \in X \backslash(A \cup B)$ if and only if $\left\{i \in I \mid x_{i}=x\right\} \in D$.

(2) $|X \backslash(A \cup B)| \leq 1$.

(3) If $x \in X \backslash(A \cup B)$, then $a<x$, for every $a \in A$, and $x<b$, for every $b \in B$.

(4) If $a \in A$ and $b \in B$, then $\left\{i \in I \mid x_{i} \in(a, b)\right\}$ belongs to $D$, hence it is not empty.

(5) If $a \in A$ and $b \in B$, then $a<b$.

(6) If $X=A \cup B$, then either $A$ has no maximum or $B$ has no minimum.

(7) If $X$ is a GO space, then $\left(x_{i}\right)_{i \in I} D$-converges to $x \in X$ if and only if one of the following (mutually exclusive) conditions holds.

(a) $\left\{i \in I \mid x_{i}=x\right\} \in D$, or

(b) $x$ is the maximum of $A$ and $x \in \bar{B}$, or

(c) $x$ is the minimum of $B$ and $x \in \bar{A}$.

(8) If $X$ is a LOTS, then $\left(x_{i}\right)_{i \in I} D$-converges to $x \in X$ if and only if either

(a) $\left\{i \in I \mid x_{i}=x\right\} \in D$, or

(b) $x$ is the maximum of $A$ and $A \cup B=X$, or

(c) $x$ is the minimum of $B$ and $A \cup B=X$.

(9) If $X$ is a GO space, then $\left(x_{i}\right)_{i \in I} D$-converges to some $x \in X$ if and only if $(A, B)$ is neither a gap, nor a pseudo-gap of $X$. 
In particular, if $X$ is a LOTS, then $\left(x_{i}\right)_{i \in I} D$-converges in $X$ if and only if $(A, B)$ is not a gap.

(10) Suppose that $X$ is a $G O$ space and that $X=A \cup B$. For $i \in I$, put

$$
O_{i}= \begin{cases}\bigcup_{a \in A}\left(x_{i}, a\right) & \text { if } x_{i} \in A, \\ \bigcup_{b \in B}\left(b, x_{i}\right) & \text { if } x_{i} \in B .\end{cases}
$$

Then $\left(x_{i}\right)_{i \in I} D$-converges to $x$ if and only if $x$ is a D-limit point of $\left(O_{i}\right)_{i \in I}$.

Proof. (1) Since $D$ is an ultrafilter, it follows that, for each $x \in X$, one and exactly one of the following sets: $\left\{i \in I \mid x<x_{i}\right\},\left\{i \in I \mid x_{i}<x\right\},\left\{i \in I \mid x_{i}=x\right\}$ belongs to $D$, since they are disjoint and their union is $I$. Hence, by the definitions, $x \notin A \cup B$ if and only if the last eventuality occurs.

(2) If $x, y \notin A \cup B$, then, by (1), both $X=\left\{i \in I \mid x_{i}=x\right\}$ and $Y=\{i \in I \mid$ $\left.x_{i}=y\right\}$ are in $D$. Since $D$ is a filter, $X \cap Y \in D$, and, since $D$ is proper, $X \cap Y \neq \emptyset$. If $i \in X \cap Y$, then $x=x_{i}=y$.

(3) If $a \in A$ and $x \notin(A \cup B)$, then both $\left\{i \in I \mid a<x_{i}\right\}$ and $\left\{i \in I \mid x_{i}=x\right\}$ belong to $D$, by (1). As above, their intersection is not empty, and this implies $a<x$. In the same way, we get that $x<b$, for every $b \in B$.

(4) If $a \in A$ and $b \in B$, then $\left\{i \in I \mid a<x_{i}\right\}$ and $\left\{i \in I \mid x_{i}<b\right\}$ belong to $D$. The intersection of the above two sets is $\left\{i \in I \mid x_{i} \in(a, b)\right\}$, therefore this set belongs to $D$, hence is nonempty.

(5) is immediate from (4).

(6) By contradiction, if $x$ is the maximum of $A$ and $y$ is the minimum of $B$, then, by (5) and since $X=A \cup B, y$ is the immediate successor of $x$. Both $\left\{i \in I \mid x<x_{i}\right\}$ and $\left\{i \in I \mid x_{i}<y\right\}$ belong to $D$, since $x \in A$ and $y \in B$. But the above two sets are disjoint, a contradiction.

(7) Suppose that $\left(x_{i}\right)_{i \in I} D$-converges to $x \in X$. By (1), (2) and the uniqueness of $D$-limits in Hausdorff spaces, either $x \notin A \cup B$ and (a) holds, or $X=A \cup B$. In this latter case, either $x \in A$ or $x \in B$.

Suppose that $x \in A$. If $x$ is not the maximum of $A$, there is $x^{\prime} \in A$ such that $x<x^{\prime}$. Then $\left(-\infty, x^{\prime}\right)$ is a neighborhood of $x$, and $\left\{i \in I \mid x^{\prime}<x_{i}\right\} \in D$, since $x^{\prime} \in A$. But then $\left\{i \in I \mid x_{i} \in\left(-\infty, x^{\prime}\right)\right\} \notin D$, contradicting $D$-convergence. Hence $x$ is the maximum of $A$. If $x \notin \bar{B}$, then, by (5) and since $A \cup B=X$, $(-\infty, x]$ is a neighborhood of $x$, hence, by $D$-convergence, $\left\{i \in I \mid x_{i} \leq x\right\} \in D$, but this contradicts $x \in A$. Next, notice that if $x \in A \cap \bar{B}$, then necessarily $x$ is the maximum of $A$, because of (5). Hence $x \in \bar{B}$.

Symmetrically, if $x \in B$, then $x$ is the minimum of $B$ and $x \in \bar{A}$. Hence one among the conditions (a)-(c) holds.

Conversely, if (a) holds, then trivially $\left(x_{i}\right)_{i \in I} D$-converges to $x$.

Suppose that (b) holds. Since $x \in A$, then, by (4), $\left\{i \in I \mid x<x_{i}<b\right\} \in D$, for every $b \in B$. Since $x \in \bar{B}$. then, for every neighborhood $U$ of $x$, there is some $b \in B$ such that $U \supseteq[x, b)$, but then $\left\{i \in I \mid x_{i} \in U\right\} \supseteq\left\{i \in I \mid x<x_{i}<b\right\} \in D$, thus $\left(x_{i}\right)_{i \in I} D$-converges to $x$.

Case (c) is proved in a symmetrical way.

Notice that (b) and (c) are mutually exclusive, since $A$ and $B$ are disjoint, by (5). Conditions (b) and (c) are also both mutually exclusive with (a) by (1).

(8) Since every LOTS is, in particular, a GO space, then Condition (7) applies. Hence it is enough to prove that, say, in every LOTS (8)(b) implies (7)(b). Indeed, if (8)(b) holds, then, since $A \cup B=X$, by (4) and (6), $x$ is an infimum of $B$, but in 
a LOTS this implies $x \in \bar{B}$, thus (7)(b) holds (notice that, by the very definition of $A$, if $A$ has a maximum, then $X \backslash A=B$ is not empty).

(9) We use the characterization given in (7). If one of (a), (b) or (c) in (7) holds, then trivially $(A, B)$ is neither a gap nor a pseudo-gap.

Conversely, suppose that $(A, B)$ is not a (pseudo-)gap. If $A \cup B \neq X$, then (7)(a) holds, for some $x \in X$, by (1). Otherwise, $A \cup B=X$. Since we are including end gaps in our definition of a gap, both $A$ and $B$ are nonempty. By (5), and since $A \cup B=X$, if neither $A$ has a maximum, nor $B$ has a minimum, then both $A$ and $B$ are open, contradicting the assumption that $(A, B)$ is not a gap. Assume that, say, $A$ has a maximum $x$. Since $A \cup B=X$, then $x$ has no immediate successor, thus $B$ has no minimum, since $x$ is the maximum of $A$. If $x \notin \bar{B}$, then $B$ would be clopen, and $(A, B)$ would be a pseudo-gap. Thus $x \in \bar{B}$ and $6(\mathrm{~b})$ holds.

(10) Suppose that $\left(x_{i}\right)_{i \in I} D$-converges to $x$. By (1), we are either in case (7)(b) or $(7)(\mathrm{c})$. Suppose, say, that we are in case (7)(b). Since $x \in \bar{B}$, then every neighborhood $U$ of $x$ contains an interval $\left[x, b^{\prime}\right]$, for some $b^{\prime} \in B$. For every $i \in I$ such that $x_{i} \in B$, there is $b \in B$ such that $b>\sup \left\{b^{\prime}, x_{i}\right\}$, by (6). Thus $b^{\prime} \in$ $\left[x, b^{\prime}\right] \cap\left(b, x_{i}\right) \subseteq U \cap O_{i}$, hence $U \cap O_{i} \neq \emptyset$. Since $x$ is the maximum of $A$, then, by (4), $\left\{i \in I \mid x_{i} \in B\right\}=\left\{i \in I \mid x<x_{i}\right\} \in D$, thus $x$ is a $D$-limit point of $\left(O_{i}\right)_{i \in I}$.

Conversely, suppose that $X=A \cup B$, and that $x$ is a $D$-limit point of $\left(O_{i}\right)_{i \in I}$. Since $X=A \cup B$, then either $\left\{i \in I \mid x_{i} \in A\right\} \in D$ or $\left\{i \in I \mid x_{i} \in B\right\} \in D$, say, the latter eventuality occurs. We first show that $x \notin B$. Indeed, if $x \in B$, then $\left\{i \in I \mid x_{i}<x\right\} \in D$ and, since $\left\{i \in I \mid x_{i} \in B\right\} \in D$, then $\left\{i \in I \mid x_{i} \in\right.$ $B \cap(-\infty, x)\} \in D$. In particular, the above set is not empty, hence there is $x^{\prime}<x$ such that $x^{\prime} \in B$. Arguing in the same way, $\left\{i \in I \mid x_{i} \in B \cap\left(-\infty, x^{\prime}\right)\right\} \in D$. But then, letting $U=\left(x^{\prime}, \infty\right)$, we have that $U$ is a neighborhood of $x$ such that $\left\{i \in I \mid U \cap O_{i}=\emptyset\right\} \in D$, since $O_{i} \subseteq\left(-\infty, x^{\prime}\right)$, whenever $x_{i} \in B \cap\left(-\infty, x^{\prime}\right)$. This contradicts the assumption that $x$ is a $D$-limit point of $\left(O_{i}\right)_{i \in I}$, hence $x \notin B$. Thus $x \in A$. Moreover, $x$ is the maximum of $A$. If not, there is some $x^{\prime} \in A$ such that $x<x^{\prime}$. Then $U=\left(-\infty, x^{\prime}\right)$ is a neighborhood of $x$ such that $U \cap O_{i}=\emptyset$, for a set of indices in $D$, namely, for $\left\{i \in I \mid x_{i} \in B\right\}$, by (5), again contradicting the assumption that $x$ is a $D$-limit point of $\left(O_{i}\right)_{i \in I}$. In view of $(7)(\mathrm{b})$, in order to finish the proof, it is enough to show that $x \in \bar{B}$. If not, $U=(-\infty, x]$ is a neighborhood of $x$, and we can get a contradiction arguing as before, since by (5) $A$ and $B$ are disjoint, $A=U=(-\infty, x]$, and $O_{i} \subseteq B$, whenever $x_{i} \in B$.

Remark 3.2. From Proposition 3.1(10) it follows easily that, for every ultrafilter $D$ and every GO space $X, D$-compactness of $X$ is equivalent to $D$-pseudocompactness of $X$. The only-if part is trivial. For the converse, suppose that $X$ is $D$-pseudocompact, and that $\left(x_{i}\right)_{i \in I}$ is a sequence of elements of $X$. If $X \neq A \cup B$, then $\left(x_{i}\right)_{i \in I} D$-converges, by 3.1(1). Otherwise, define $\left(O_{i}\right)_{i \in I}$ as in (10). It is enough to show that $\left\{i \in I \mid O_{i} \neq \emptyset\right\} \in D$, since then we can apply $D$-pseudocompactness to $\left(O_{i}\right)_{i \in I}$ and use (10). Since $X=A \cup B$, then either $\left\{i \in I \mid x_{i} \in A\right\} \in D$, or $\left\{i \in I \mid x_{i} \in B\right\} \in D$. Say the latter case occurs, then, by the definition, $B$ has no minimum, hence, for every $i \in I$ such that $x_{i} \in B$, we have $O_{i}=B \cap\left(-\infty, x_{i}\right) \neq \emptyset$. Hence the $O_{i}$ 's are nonempty for a set of indices in $D$, namely, a set containing $\left\{i \in I \mid x_{i} \in B\right\}$. 
However, much more can be said about the connections among $D$-compactness, $D$-pseudocompactness and other properties of a GO space $X$. This is the main theme of the next section.

\section{D-compactness, D-pseudocompactness, Gaps, Products, etc.}

To state the results of the present section in their full generality, we need some more definitions.

An ultrafilter $D$ over some set $I$ is $\lambda$-descendingly complete if every $\subseteq$-decreasing sequence $\left(Z_{\alpha}\right)_{\alpha \in \lambda}$ of sets in $D$ has intersection still in $D$. The ultrafilter $D$ is $\lambda$ decomposable if there is a function $f: I \rightarrow \lambda$ such that $f^{-1}(X) \notin D$, for every $X \subseteq \lambda$ such that $|X|<\lambda$. Clearly, if $\lambda$ is an infinite cardinal, a $\lambda$-decomposable ultrafilter is not $\lambda$-descendingly complete: just consider $Z_{\alpha}=f^{-1}([\alpha, \lambda))$. If $\lambda$ is regular, the converse holds. If $\lambda$ is regular and $D$ is not $\lambda$-descendingly complete, as witnessed by $\left(Z_{\alpha}\right)_{\alpha \in \lambda}$, the following function $f$ witnesses that $D$ is $\lambda$-decomposable. Define $f$ by letting $f(i)$ be the smallest $\alpha$ such that $i \notin Z_{\alpha}$, if such an $\alpha<\lambda$ exists; $f(i)$ is unimportant and can be arbitrary if $i \in \bigcap_{\alpha \in \lambda} Z_{\alpha}$, since we are assuming that $\bigcap_{\alpha \in \lambda} Z_{\alpha} \notin D$. Thus, for infinite regular cardinals, $\lambda$-decomposability is equivalent to the negation of $\lambda$-descending completeness. Let us also mention that there is another notion equivalent to $\lambda$-decomposability, for $\lambda$ an infinite regular cardinal, that is, $(\lambda, \lambda)$-regularity. We shall make only a limited use of $(\lambda, \lambda)$ regularity here (see Sections 5] and 6), but we should warn the reader that theorems about $\lambda$-descending (in)completeness or $\lambda$-decomposability are frequently stated in equivalent forms in terms of regularity, or vice versa. A full discussion is given in Lip10; see Section 1 there and, in particular, Properties 1.1(xi) and Consequence 1.2 .

For an ultrafilter $D$, let $K_{D}=\{\nu \geq \omega \mid D$ is $\nu$-decomposable $\}$. Many problems are still open about the possible values $K_{D}$ can assume, and solutions to such problems are heavily dependent on the universe of set theory one is working in. For example, in certain models of set theory, $K_{D}$ is always an interval of cardinals, with bottom element $\omega$; on the other hand, there are models in which $K_{D}$ is a rather sparse set of cardinals. We refer to the comments after Problem 6.8 in Lip10 for further information and details, or to the remarks before Corollary 6.4 here, where we show that such set theoretical problems affect the behavior under products of certain compactness properties of GO spaces. In particular, certain statements turn out to be independent from the usual axioms for set theory. In this paper we shall be mainly concerned with regular cardinals in $K_{D}$, hence we shall establish the special notation $K_{D}^{R e g}$ for the set of such cardinals. Namely, $K_{D}^{R e g}=\{\nu \geq \omega \mid \nu$ is regular and $D$ is $\nu$-decomposable $\}$.

A topological space is $[\nu, \lambda]$-compact if every open cover of cardinality $\leq \lambda$ has a subcover of cardinality $<\nu$. It is a classical result by Alexandroff and Urysohn AU29] that if $\nu$ is a regular cardinal, then $[\nu, \nu]$-compactness of a topological space $X$ is equivalent to $\mathrm{CAP}_{\nu}$, which is the property asserting that every subset of $X$ of cardinality $\nu$ has a complete accumulation point. Initial $\lambda$-compactness is $[\omega, \lambda]$ compactness, and, again by [AU29], it is equivalent to $[\nu, \nu]$-compactness, for every cardinal $\nu \leq \lambda$, equivalently, for every regular cardinal $\nu \leq \lambda$. As usual, by $[\nu, \lambda]$ we shall also denote the interval of all cardinals $\mu$ such that $\nu \leq \mu \leq \lambda$. We hope that the partially overlapping notation will cause no confusion. 
A topological space is weakly $[\nu, \lambda]$-compact if every open cover of cardinality $\leq \lambda$ has a subfamily of cardinality $<\nu$ with dense union. Weak initial $\lambda$-compactness is weak $[\omega, \lambda]$-compactness. The above notions are frequently named using very disparate terminology. Weak $[\nu, \lambda]$-compactness has sometimes been called weak $\lambda-\nu$ compactness, and we used still different terminology in [Lip12, where we called it $\mathcal{O}$ $[\nu, \lambda]$-compactness. When $\nu$ is a regular cardinal, weak $[\nu, \nu]$-compactness is equivalent to a notion called pseudo- $(\kappa, \kappa)$-compactness. Weak initial $\omega$-compactness is equivalent to faint compactness, and equivalent to pseudocompactness in the class of Tychonoff spaces. Weak initial $\lambda$-compactness has been called almost $\lambda$ compactness, or weak- $\lambda$ - $\aleph_{0}$-compactness by some authors. See [Lip11b, Remark 3] for further details and references.

If $\lambda$ is an infinite cardinal, a sequence $\left(x_{\gamma}\right)_{\gamma<\lambda}$ of elements of a topological space converges to some point $x$ if, for every neighborhood $U$ of $x$, there is $\gamma<\lambda$ such that $x_{\gamma^{\prime}} \in U$, for every $\gamma^{\prime}>\gamma$.

If $(A, B)$ is a gap of some GO space, we say that that $\lambda$ is a type of $(A, B)$ if either $A$ has cofinality $\lambda$, or $B$ has coinitiality $\lambda$ (thus a gap has at most two types). If $(A, B)$ is a pseudo-gap, say, $B$ having a minimum, the type of $(A, B)$ is the cofinality of $A$, and, symmetrically, the coinitiality of $B$, if $A$ has a maximum.

The above two notions are related as follows. If $\left(x_{\gamma}\right)_{\gamma<\lambda}$ is a non converging strictly increasing sequence of elements of a GO space $X$, then, letting $A=\{x \in$ $X \mid x<x_{\gamma}$ for some $\left.\gamma<\lambda\right\}$, and $B=X \backslash A$, we have that $(A, B)$ is a (pseudo-)gap having type $\lambda$. A symmetrical statement holds for strictly decreasing sequences. Conversely, given a (pseudo-)gap $(A, B)$ having type $\lambda$, we obtain a strictly monotone non converging sequence $\left(x_{\gamma}\right)_{\gamma<\lambda}$, by considering either a cofinal subset of $A$ or a coinitial subset of $B$.

Theorem 4.1. Suppose that $D$ is an ultrafilter over some set $I$, and recall that $K_{D}^{R e g}$ is the set of those infinite regular cardinals $\nu$ such that $D$ is $\nu$-decomposable. For every $G O$ space $X$, the following conditions are equivalent.

(1) $X$ is D-compact.

(2) $X$ is $[\nu, \nu]$-compact, for every cardinal $\nu \in K_{D}^{R e g}$.

(3) For every cardinal $\nu \in K_{D}^{R e g}$, and every strictly increasing (resp., strictly decreasing) $\nu$-indexed sequence of elements of $X$, the sequence has a supremum (resp., an infimum) to which it converges.

(4) $X$ has no (pseudo-)gap of type belonging to $K_{D}^{\text {Reg }}$.

(5) $X$ is D-pseudocompact.

(6) $X$ is weakly $[\nu, \nu]$-compact, for every cardinal $\nu \in K_{D}^{R e g}$.

(7) For every cardinal $\nu \in K_{D}^{R e g}$, and every sequence $\left(O_{\gamma}\right)_{\gamma<\nu}$ of open nonempty sets of $X$, there is $x \in X$ such that $\left|\left\{\gamma<\nu \mid O_{\gamma} \cap U \neq \emptyset\right\}\right|=\nu$, for every neighborhood $U$ of $x$.

In the above condition we can equivalently require either that the $O_{\gamma}$ 's are pairwise disjoint, or that $O_{\gamma} \subset O_{\gamma^{\prime}}$, for $\gamma>\gamma^{\prime}$.

(8) $X$ is $D^{\prime}$-compact, for every ultrafilter $D^{\prime}$ such that $K_{D^{\prime}}^{\text {Reg }} \subseteq K_{D}^{R e g}$.

(9) $X$ is $D^{\prime}$-pseudocompact, for every ultrafilter $D^{\prime}$ such that $K_{D^{\prime}}^{R e g} \subseteq K_{D}^{R e g}$.

Proof. $(1) \Rightarrow(2)$ It is enough to show that if $\nu$ is regular and $D$ is $\nu$-decomposable, then every $D$-compact topological space is $[\nu, \nu]$-compact. This is essentially a classical result (which holds for every topological space), due to GS75 in the case $\nu=\omega$, and to [Sak78] in the general case. See also Cai99 and Lip12 for other 
versions and generalizations. Briefly, and using the equivalent reformulation of $[\nu, \nu]$-compactness in terms of complete accumulation points, if $Y \subseteq X$ and $|Y|=\nu$, enumerate $Y$ as $\left\{y_{\gamma} \mid \gamma \in \nu\right\}$. If $f: I \rightarrow \nu$ witnesses the $\nu$-decomposability of $D$, then, by $D$-compactness, the sequence $\left(y_{f(i)}\right)_{i \in I}$ has some $D$-limit point, which is easily seen to be also a complete accumulation point of $Y$.

$(2) \Rightarrow(3)$ is almost immediate. Indeed, if, by contradiction, $\left(x_{\gamma}\right)_{\gamma<\nu}$ is, say, strictly increasing, then $O=X \backslash \bigcup_{\gamma<\nu}\left(-\infty, x_{\gamma}\right)$ is open. Then the family containing $O$ and $\left(-\infty, x_{\gamma}\right)$, for $\gamma<\nu$, is an open cover by $\nu$ sets, but no subfamily by $<\nu$ sets is a cover, since $\nu$ is regular.

The equivalence of (3) and (4) should be clear from the remarks shortly before the statement of the theorem.

The proof of $(4) \Rightarrow(1)$ is the key argument in the proof of the theorem, and uses in an essential way the assumption that we are in a GO space. Suppose by contradiction that (1) fails and let $\left(x_{i}\right)_{i \in I}$ be a sequence which does not $D$-converge. Let $A$ and $B$ be defined as in the statement of Proposition 3.1. By condition (9) in the same proposition, $(A, B)$ is either a gap or a pseudo-gap of $X$, in particular, $A \cup B=X$. Hence either $\left\{i \in I \mid x_{i} \in A\right\} \in D$, or $\left\{i \in I \mid x_{i} \in B\right\} \in D$. Suppose, say, that the latter occurs. Then by the assumption that $\left\{i \in I \mid x_{i} \in B\right\} \in D$, by the definition of $B$, and since $D$ is a filter, we get that, for every $b \in B$, $X_{b}=\left\{i \in I \mid x_{i} \in(-\infty, b) \cap B\right\} \in D$. Notice that it follows that $B$ has no minimum, since each $X_{b}$ is nonempty. Choose a strictly decreasing coinitial sequence $\left(b_{\gamma}\right)_{\gamma \in \nu}$ in $B$. Then $\nu$ is a type of the (pseudo-)gap $(A, B)$, and $\left(X_{b_{\gamma}}\right)_{\gamma \in \nu}$ witnesses that $D$ is not $\nu$-descendingly complete, since $\bigcap_{\gamma \in \nu} X_{b_{\gamma}}=\left\{i \in I \mid x_{i} \notin B\right\} \notin D$. Thus $\nu \in K_{D}^{R e g}$, by the equivalence mentioned after the definition of decomposability, and since $\nu$ is regular, being the coinitiality of $B$. Thus $(A, B)$ has type $\nu \in K_{D}^{R e g}$, contradicting (4).

So far, we have proved the equivalence of (1)-(4).

$(1) \Rightarrow(5)$ is trivial.

$(5) \Rightarrow(6)$ is similar to $(1) \Rightarrow(2)$, by considering a sequence of nonempty open sets of $X$, rather than a sequence of elements of $X$. Full details can be found in Lip12, Fact 6.1, Corollary 4.6 and condition (d) in Theorem 4.4], by taking $\mathcal{F}=\mathcal{O}$ there.

The equivalence of (6) with the first statement in (7) is true in every topological space, and is similar to the equivalence of $[\nu, \nu]$-compactness with $\mathrm{CAP}_{\nu}$ (recall that we are assuming that $\nu$ is regular). Details can be found again in Lip12, by taking $\mathcal{F}=\mathcal{O}$ in Theorem $4.4(\mathrm{a}) \Leftrightarrow(\mathrm{c})$ there.

For GO spaces, $(7) \Rightarrow(3)$ is a standard argument. Say, $\left(x_{\gamma}\right)_{\gamma \in \nu}$ is strictly increasing. Put $O_{\gamma}=\bigcup_{\eta>\gamma}\left(x_{\gamma}, x_{\eta}\right)$. Then if $x$ is such that $\mid\left\{\gamma<\nu \mid O_{\gamma} \cap U \neq\right.$ $\emptyset\} \mid=\nu$, for every neighborhood $U$ of $x$, then necessarily $\left(x_{\gamma}\right)_{\gamma \in \nu}$ converges to $x$. Here the $O_{\gamma}$ 's are strictly decreasing with respect to inclusion.

If we want the $O_{\gamma}$ 's to be pairwise disjoint, then, for $\gamma=\alpha+n$ with $\alpha=0$ or $\alpha$ limit, take $O_{\gamma}=\left(x_{\alpha+2 n}, x_{\alpha+2 n+2}\right)$.

Hence (1)-(7) are all equivalent, for every ultrafilter $D$ and every GO space $X$.

$(2) \Rightarrow(8)$ Suppose that $D^{\prime}$ is such that $K_{D^{\prime}}^{R e g} \subseteq K_{D}^{R e g}$. If (2) holds, then, since $K_{D^{\prime}}^{R e g} \subseteq K_{D}^{R e g}, X$ is $[\nu, \nu]$-compact, for every cardinal $\nu \in K_{D^{\prime}}^{R e g}$. Applying the equivalence of (1) and (2) in the case of the ultrafilter $D^{\prime}$, we get that $X$ is $D^{\prime}$-compact.

$(8) \Rightarrow(1)$ follows trivially by taking $D=D^{\prime}$. 
The equivalence of (9) with, say, (6) and (5) is similar.

Given a family $\left(X_{j}\right)_{j \in J}$ of GO spaces, the product $\prod_{j \in J} X_{j}$ is not necessarily a GO space; however, it is a topological space, when endowed with the (Tychonoff) product topology. $\prod_{j \in J} X_{j}$ can also be given the structure of a partially ordered set, by letting the relation $\mathbf{x} \leq \mathbf{y}$ hold in $\prod_{j \in J} X_{j}$ if and only if it holds componentwise. The next corollary deals with the above structures on $\prod_{j \in J} X_{j}$. It shows that if each $X_{j}$ is a GO space, then many properties of $\prod_{j \in J} X_{j}$ are determined by the corresponding properties of the $X_{j}$ 's.

To avoid trivial exceptions, we shall always assume that all factors in a product are nonempty.

Corollary 4.2. Suppose that $D$ is an ultrafilter over some set I. For every family $\left(X_{j}\right)_{j \in J}$ of $G O$ spaces, the following conditions are equivalent.

(a) For every $j \in J$, the GO space $X_{j}$ satisfies one (and hence all) of the conditions in Theorem 4.1 .

(b) The topological space $\prod_{j \in J} X_{j}$ satisfies all of the conditions (1)-(2),(5)-(9) in Theorem 4.1.

(c) The topological space $\prod_{j \in J} X_{j}$ satisfies one of the conditions (1)-(2),(5)-(9) in Theorem 4.1.

(d) For every cardinal $\nu \in K_{D}^{R e g}$, every monotone $\nu$-indexed sequence in $\prod_{j \in J} X_{j}$ converges.

Proof. (a) $\Rightarrow$ (b) If each $X_{j}$ satisfies any one of the conditions in Theorem 4.1 , then, by the very same theorem, $X_{j}$ is $D$-compact. By an easy and classical property of $D$-compactness Ber70, GS75, Sak78, every product of $D$-compact spaces is still $D$-compact, hence $\prod_{j \in J} X_{j}$ is still $D$-compact. Now notice that the proof that (1) implies any one of the conditions (1)-(2),(5)-(7) in Theorem 4.1 holds for an arbitrary topological space, not only for GO spaces. Moreover, if each $X_{j}$ is $D$-compact, then, by Theorem $4.1(1) \Rightarrow(8)$, each $X_{j}$ is $D^{\prime}$-compact, for every ultrafilter $D^{\prime}$ such that $K_{D^{\prime}}^{R e g} \subseteq K_{D}^{R e g}$. Again since $D^{\prime}$-compactness is preserved under products, $\prod_{j \in J} X_{j}$ is $D^{\prime}$-compact, thus condition 4.1(8) holds for $\prod_{j \in J} X_{j}$. The proof that (9) holds for $\prod_{j \in J} X_{j}$ is similar, since $D^{\prime}$-pseudocompactness is preserved under products, too, GS75.

(b) $\Rightarrow$ (c) is trivial.

(c) $\Rightarrow$ (a) If $\prod_{j \in J} X_{j}$ satisfies any one of the conditions (1)-(2),(5)-(9), then trivially each $X_{j}$ satisfies the same condition, hence (a) holds.

(a) $\Rightarrow$ (d) By Theorem 4.1, if (a) holds, then condition (3) in Theorem 4.1 holds, for every $j \in J$. Suppose that $\left(\mathbf{x}_{\gamma}\right)_{\gamma \in \nu}$ is a monotone, say, increasing, sequence in $\prod_{j \in J} X_{j}$. Since $\nu$ is a regular cardinal, for every $j \in J$, the projection $\left(x_{j, \gamma}\right)_{\gamma \in \nu}$ of $\left(\mathbf{x}_{\gamma}\right)_{\gamma \in \nu}$ into $X_{j}$ is either eventually constant, or has a strictly increasing subsequence of order type $\nu$. In both cases, $\left(x_{j, \gamma}\right)_{\gamma \in \nu}$ converges in $X_{j}$. This is trivial in the former case; in the latter case, the strictly increasing subsequence converges, by condition (3) in Theorem 4.1, and then also $\left(x_{j, \gamma}\right)_{\gamma \in \nu}$ converges (to the same point), since it is increasing. Now it is trivial to see that a sequence in a product converges if and only if each projection converges, hence $\left(\mathbf{x}_{\gamma}\right)_{\gamma \in \nu}$ converges in $\prod_{j \in J} X_{j}$.

(d) $\Rightarrow$ (a) If (d) holds, then trivially each $X_{j}$ satisfies condition (3) in Theorem 4.1, thus (a) holds. 


\section{THE PARTICULAR CASE OF INITIAL COMPACTNESS}

For LOTS, the equivalence of conditions (2), (4) and (10) in the next corollary has first been proved, under different terminology and with further equivalences, in GFW70, Theorem 3]. For GO spaces the corollary appears in Lip13.

Recall that an ultrafilter $D$ over $\lambda$ is regular if there is a family $\left(Z_{\alpha}\right)_{\alpha \in \lambda}$ of members of $D$ such that the intersection of any infinite subfamily is empty (this is also called $(\omega, \lambda)$-regularity). It is a standard application of the Axiom of Choice, or just the Prime Ideal Theorem, to show that, for every infinite cardinal $\lambda$, there exists a regular ultrafilter $D$ over $\lambda$. See, e. g., Chang and Keisler CK90, Proposition 4.3.5]. For such an ultrafilter, it is easy to show that $\nu \in K_{D}$, for every regular $\nu \leq \lambda$ : see [Lip10, Properties 1.1(xii)] (in fact, one actually has that $K_{D}=[\omega, \lambda]$, by [Lip10, Properties 1.1(1) and the last paragraph in Remark 1.5(b)], but we shall not need this here). It follows that $K_{D^{\prime}}^{R e g} \subseteq K_{D}^{R e g}$, for every regular ultrafilter over $\lambda$, and every ultrafilter $D^{\prime}$ over any set of cardinality $\leq \lambda$.

Corollary 5.1. For every infinite cardinal $\lambda$, and every $G O$ space $X$, the following conditions are equivalent.

(1) $X$ is D-compact, for some regular ultrafilter over $\lambda$.

(2) $X$ is initially $\lambda$-compact.

(3) For every infinite regular cardinal $\nu \leq \lambda$, and every strictly increasing (resp., strictly decreasing) $\nu$-indexed sequence of elements of $X$, the sequence has a supremum (resp., an infimum) to which it converges.

(4) $X$ has no (pseudo-)gap having type $\leq \lambda$.

(5) $X$ is D-pseudocompact, for some regular ultrafilter over $\lambda$.

(6) $X$ is weakly initially $\lambda$-compact.

(7) For every infinite (equivalently, every infinite regular) cardinal $\nu \leq \lambda$, and every sequence $\left(O_{\gamma}\right)_{\gamma<\nu}$ of open nonempty sets of $X$, there is $x \in X$ such that $\left|\left\{\gamma<\nu \mid O_{\gamma} \cap U \neq \emptyset\right\}\right|=\nu$, for every neighborhood $U$ of $x$.

In the above condition we can equivalently require either that the $O_{\gamma}$ 's are pairwise disjoint, or that $O_{\gamma} \subset O_{\gamma^{\prime}}$, for $\gamma>\gamma^{\prime}$.

(8) $X$ is D-compact, for every ultrafilter $D$ over any set of cardinality $\leq \lambda$.

(9) $X$ is D-pseudocompact, for every ultrafilter $D$ over any set of cardinality $\leq \lambda$.

(10) $X$ is $\lambda$-bounded, that is, every subset of cardinality $\leq \lambda$ has compact closure.

Proof. We are first giving the proof as a consequence of Theorem 4.1. As we mentioned in the introduction, a more direct proof (which nevertheless uses essentially the same ideas) can be found in Lip13. We then sketch an alternative proof which goes along with some arguments in GFW70.

Most of the equivalences in the corollary are the particular cases of Theorem 4.1 applied when $D$ is a regular ultrafilter over $\lambda$. This is the case for conditions (1), (3)-(5), (7) reg, (8) and (9), by the remarks before the statement of the corollary, and where by $(7)_{\text {reg }}$ we denote condition (7) restricted to regular $\nu$ 's.

As far as (2) is concerned, it is standard and already proved in AU29] that initial $\lambda$-compactness is equivalent to $[\nu, \nu]$-compactness for every regular $\nu \leq \lambda$, so we get the equivalence with condition (2) in Theorem 4.1 in the case when $D$ is regular over $\lambda$. Alternatively, (1) $\Rightarrow(2)$ in the present corollary follows immediately from Cai99, Theorem 3.4]. Notice that Cai99, following standard use in the model 
theoretical setting, uses a notation in which the order of the cardinals is reversed, both in the definition of $[\mu, \nu]$-compactness and of $(\omega, \nu)$-regularity.

In contrast with (2), it is not always necessarily the case that (for topological spaces in general) weak $[\nu, \nu]$-compactness for every regular $\nu \leq \lambda$ implies weak initial $\lambda$-compactness. This is Remark 30 in Lip11b, relying on an example by García-Ferreira GF99, which in turn builds on a construction by Kanamori Kan86. However, (5) implies (6) by Lip11b, Corollary 15], (6) implies (7) by Lip11a, Theorem 1] and Retta [Ret93, Theorem 3(d)], and (7) implies (7) reg trivially, hence, for GO spaces, they are all equivalent, since we have already proved that for GO spaces (5) and (7) reg are equivalent.

Finally, the equivalence of (8) and (10) holds for every Hausdorff regular space, Sak78, Theorems 5.3 and 5.4] (recall that it can be proved that every GO space is regular). Our first proof of the corollary is thus complete.

An alternative proof of (4) implies (10) goes as follows. Suppose that $Y \subseteq X$, and $|Y| \leq \lambda$. The closure $\bar{Y}$ of $Y$ has no (pseudo-)gap of type $\leq \lambda$, since otherwise it would extend to a (pseudo-)gap of $X$ having the same type. Moreover, $\bar{Y}$ has no subset of order type or reversed order type $>\lambda$, since then one could construct a subset of $Y$ having the same order type, but this is impossible, since $|Y| \leq \lambda$. In conclusion, $\bar{Y}$ has no (pseudo-)gap at all, but this implies that it is compact, by a well-known result, e. g., Nagata Nag85, Theorem VIII.2].

Then an alternative proof of the corollary is obtained by the following chains of implications $(4) \Rightarrow(10) \Rightarrow(8) \Rightarrow(1) \Rightarrow(2) \Rightarrow(3) \Rightarrow(4)$ and $(4) \Rightarrow(10) \Rightarrow(8) \Rightarrow$ $(9) \Rightarrow(5) \Rightarrow(6) \Rightarrow(7) \Rightarrow(4)$, which are either trivial, or proved before.

Corollary 5.2. Suppose that $X$ is a product of topological spaces and that all factors but at most one are GO spaces. Then the following hold.

(1) $X$ is initially $\lambda$-compact if and only if each factor is initially $\lambda$-compact.

(2) $X$ is weakly initially $\lambda$-compact if and only if each factor is weakly initially $\lambda$-compact.

Proof. (1) An implication is trivial. For the other direction, by the equivalence of (2) and (10) in Corollary [5.1, all but at most one factor are $\lambda$-bounded. A product of regular $\lambda$-bounded spaces is still $\lambda$-bounded [GFW70, Lemma 4], or [Ste84, Theorem 5.7 and implications (1), (1') in Diagram 3.6], and a product of a $\lambda$-bounded space with an initially $\lambda$-compact space is initially $\lambda$-compact Ste84, Theorem 5.2 and implications (1), (2) in Diagram 3.6]. Hence (1) follows by first grouping together the GO spaces, and then, in case, multiplying their product with the possibly non GO factor. Alternatively, one can use an argument parallel to the one we are going to give for (2).

(2) Again, an implication is trivial. For the other direction, by the equivalence of (6) and (9) in Corollary 5.1, all but at most one factor are $D$-pseudocompact, for every ultrafilter $D$ over any set of cardinality $\lambda$. Since $D$-pseudocompactness is preserved under products GS75, we have that all but at most one factor are $D$-pseudocompact, for every ultrafilter $D$ over any set of cardinality $\lambda$. Since the (possible) remaining factor is weakly initially $\lambda$-compact, by assumption, (2) follows from the next lemma (which, in some form or another, is probably folklore).

Lemma 5.3. If the topological space $X$ is D-pseudocompact, for every ultrafilter $D$ over $\lambda$, and the topological space $Y$ is weakly initially $\lambda$-compact, then $X \times Y$ is weakly initially $\lambda$-compact. 
PAOLO LIPPARINI

Proof. Let $S_{\omega}(\lambda)$ denote the set of all finite subsets of $\lambda$. By Lip11b, Theorem 10], a topological space is weakly initially $\lambda$-compact if and only if, for every sequence $\left\{O_{Z} \mid Z \in S_{\omega}(\lambda)\right\}$ of nonempty open sets, there exists an ultrafilter D over $S_{\omega}(\lambda)$ such that both $\left\{Z \in S_{\omega}(\lambda) \mid \alpha \in Z\right\} \in D$, for every $\alpha \in \lambda$, and $\left\{O_{Z} \mid Z \in S_{\omega}(\lambda)\right\}$ has a $D$-limit point.

So let $\left\{O_{Z} \mid Z \in S_{\omega}(\lambda)\right\}$ be a sequence of nonempty open sets in $X \times Y$ : it is enough to find some $D$ as above. Without loss of generality, we can suppose that $O_{Z}=U_{Z} \times V_{Z}$, for every $Z \in S_{\omega}(\lambda)$. Since $Y$ is weakly initially $\lambda$-compact, by the quoted theorem, there exists an ultrafilter $\mathrm{D}$ as above such that $\left\{V_{Z} \mid Z \in S_{\omega}(\lambda)\right\}$ has a $D$-limit point $y$. Since $\left|S_{\omega}(\lambda)\right|=\lambda$, then $X$ is $D$-pseudocompact, hence also $\left\{U_{Z} \mid Z \in S_{\omega}(\lambda)\right\}$ has a $D$-limit point $x$. But then $(x, y)$ is a $D$-limit point of $\left\{O_{Z} \mid Z \in S_{\omega}(\lambda)\right\}$.

See [GFW70, Corollary on p. 203] for other results related to Corollary [5.2 See GFS97 for further results in the particular case of countable compactness, that is, $\lambda=\omega$.

\section{THE IMPACT OF SET THEORY}

In view of Theorem $4.1(2) \Leftrightarrow(4)$, one would be tempted to conjecture that, for every infinite regular cardinal $\nu$, a GO space $X$ is $[\nu, \nu]$-compact if and only if $X$ ha no (pseudo-)gap of type $\nu$. However this is false: just consider $\mathbb{R}$ with the usual order, and with the discrete topology. The counterexample can be easily turned into a LOTS: let $X$ be the product $\mathbb{R} \times \mathbb{Z}$, with the lexicographic order. The induced topology on $X$ is the discrete one and, since $|X|=2^{\omega}$, then $X$ is not $[\nu, \nu]$-compact, for every $\nu \leq 2^{\omega}$. On the other hand, $X$ has only gaps of type $\omega$, thus, taking $\nu=\omega_{1}$ (or any $\nu$ with $\omega<\nu \leq 2^{\omega}$-of course, this is significant only if the Continuum Hypothesis fails), we get that having no gap of type $\nu$ does not necessarily imply $[\nu, \nu]$-compactness. Indeed, the equivalence holds only for a very special class of cardinals. It can be shown that a regular cardinal $\nu>\omega$ is weakly compact if and only if, for every GO space (equivalently, LOTS) $X,[\nu, \nu]$-compactness of $X$ is equivalent to $X$ having no (pseudo-)gap of type $\nu$.

Put in another way, an equivalence like $(2) \Leftrightarrow(4)$ in Theorem 4.1 can hold only if considered simultaneously for all $\nu$ in some appropriate class $H$ of regular cardinals. Theorem 4.1 shows that the equivalence holds in case $H$ can be realized as $K_{D}^{R e g}$, for some ultrafilter $D$. In this sense, the existence of a regular ultrafilter over $\lambda$ can be seen as a fact "responsible behind the scene" for the equivalence of initial $\lambda$-compactness with the nonexistence of (pseudo-)gaps of type $\leq \lambda$. As we mentioned, we have a proof that if $H=\{\nu\}$ is a singleton, then the equivalence 4.1 $(2) \Leftrightarrow(4)$ (with $H$ in place of $K_{D}^{R e g}$ there) holds if and only if either $\nu=\omega$, or $\nu$ is weakly compact.

The above considerations suggest the following definition. If $H$ a class of infinite cardinals, let us say that a topological space is $H$-compact if it is $[\nu, \nu]$-compact, for every $\nu \in H$. Thus the equivalence $(2) \Leftrightarrow(4)$ in Theorem 4.1 asserts that if $H=K_{D}^{R e g}$, for some ultrafilter $D$, then a GO space $X$ is $H$-compact if and only if $X$ has no (pseudo-)gap of type in $H$. If we strengthen the above conditions by asking forms of preservation under products, we get that the corresponding equivalence holds exactly for those classes $H$ which can be expressed as unions of classes of the form $K_{D}^{R e g}$. This is the content of the next corollary, which also shows that in 
many cases, say, when $H$ is finite, the equivalence holds if and only if $H=K_{D}^{R e g}$, for some ultrafilter $D$. Thus, for GO spaces, preservation of $H$-compactness under products is highly dependent on set theory, as we shall discuss shortly after the proof of the corollary.

Recall that an ultrafilter $D$ is $(\lambda, \mu)$-regular if there is a set of $\mu$ members of $D$ such that the intersection of any $\lambda$ of them is empty. We refer again to Lip10 for more information about regularity of ultrafilters. Here regularity shall be used in connection with results from [Cai99, asserting that, roughly, $(\lambda, \mu)$-regular ultrafilters are standard witnesses for $[\lambda, \mu]$-compactness of products of topological spaces.

Let Reg denote the class of all infinite regular cardinals. An interval of regular cardinals is a set of the form Reg $\cap[\lambda, \mu]$, for certain cardinals $\lambda$ and $\mu$. Notice that we allow $\mu$ to be singular. If $H$ is a set of cardinals, and $F$ is a finite union of intervals of regular cardinals, we say that $H$ includes the cofinalities of the extremes of $F$ if $F$ can be represented as $F=\operatorname{Reg} \cap \bigcup_{p \in P}\left[\lambda_{p}, \mu_{p}\right]$, with $P$ finite, and in such a way that $\operatorname{cf} \mu_{p} \in H$, for every $p \in P$ (of course, in case $H=F$ this is relevant only when some $\mu_{p}$ is singular).

Corollary 6.1. Suppose that $H$ is a class of infinite regular cardinals. Then the following statements are equivalent.

(1) Every product of a family of $H$-compact $G O$ spaces is still $H$-compact.

(2) Same as (1), restricted to spaces which are regular cardinals with the order topology.

(3) For every $\nu \in H$ there is an ultrafilter $D_{\nu}$ such that $\nu \in K_{D_{\nu}}$ and $K_{D_{\nu}}^{\text {Reg } \subseteq}$ $H$.

$\left(3^{\prime}\right)$ There is a class $\mathcal{D}$ of ultrafilters such that $H=\bigcup_{D \in \mathcal{D}} K_{D}^{R e g}$.

(4) Every product of GO spaces without (pseudo-)gaps of type in $H$ is $H$ compact.

(5) More generally, if $Y$ is a product of $G O$ spaces, and each factor of $Y$ satisfies at least one of conditions (2)-(4), (6)-(7) in Theorem 4.1, with $K_{D}^{\text {Reg }}$ there replaced by $H$, then $Y$ is $H$-compact.

$\left(5^{\prime}\right)$ If $Y$ is a product of GO spaces, and each factor of $Y$ satisfies all the conditions (2)-(4), (6)-(7) in Theorem 4.1, with $K_{D}^{\text {Reg }}$ there replaced by $H$, then $Y$ is $H$-compact.

(6) For every finite $F \subseteq H$, there is an ultrafilter $D_{F}$ such that $F \subseteq K_{D_{F}}^{R e g} \subseteq H$.

(7) More generally, for every set $F \subseteq H$ such that $F$ is a finite union of intervals of regular cardinals, and $H$ includes the cofinalities of the extremes of $F$, there is an ultrafilter $D_{F}$ such that $F \subseteq K_{D_{F}}^{R e g} \subseteq H$.

Suppose in addition that $H$ is finite, or, more generally, that $H$ is a finite union of intervals of regular cardinals and $H$ includes the cofinalities of its extremes. Then the preceding conditions are also equivalent to the following one.

(8) There is an ultrafilter $D$ such that $K_{D}^{\text {Reg }}=H$.

Proof. $(1) \Rightarrow(2)$ is trivial.

$(2) \Rightarrow(3)$ Let $\mathcal{T}$ be the class of the regular cardinals which are not in $H$. Notice that every member of $\mathcal{T}$ is $[\nu, \nu]$-compact, for every $\nu \in H$. By (2), every product of members of $\mathcal{T}$, too, is $[\nu, \nu]$-compact, for every $\nu \in H$. For every $\nu \in H$, by Sak78 or Cai99. Theorem 3.4], there exists a $\nu$-decomposable (equivalently, $(\nu, \nu)$-regular, since $\nu$ is regular) ultrafilter $D_{\nu}$ such that every member of $\mathcal{T}$ is $D_{\nu}$-compact. It 
is easy to see that if $\kappa$ is a regular cardinal, $D$ is an ultrafilter, and the space $\kappa$ is $D$-compact, then $D$ is $\kappa$-descendingly complete (see the proof of Lip96, Proposition $1]$, though the result is stated with different terminology), equivalently, $D$ is not $\kappa$-decomposable. In the case at hand, this shows that if $\kappa$ is regular and does not belong to $H$, then $\kappa \notin K_{D_{\nu}}$. This means exactly that $K_{D_{\nu}}^{R e g} \subseteq H$. Since $D_{\nu}$ is $\nu$-decomposable, then $\nu \in K_{D_{\nu}}$, hence $D_{\nu}$ witnesses (3).

$\left(3^{\prime}\right)$ is a restatemetn of $(3)$.

$(3) \Rightarrow(4)$ We have to show that every product $Y$ as in (4) is $[\nu, \nu]$-compact, for every $\nu \in H$. So let $\nu \in H$, and let $D_{\nu}$ be given by (3). Since $K_{D_{\nu}}^{R e g} \subseteq H$, then no factor of $Y$ has a (pseudo-)gap in $K_{D_{\nu}}^{R e g}$. By Theorem 4.1 (4) $\Rightarrow$ (1) applied to $D_{\nu}$, each factor of $Y$ is $D_{\nu}$-compact, hence also $Y$ is $D_{\nu}$-compact. Hence $Y$ is $[\nu, \nu]$-compact, since $\nu \in K_{D_{\nu}}^{R e g}$ and, as we mentioned, 4.1 (1) $\Rightarrow(2)$ holds for every topological space. (Of course, we are repeating here some arguments from the proof of Corollary 4.2, and the implication can be also obtained as a consequence of 4.2).

$(4) \Rightarrow(1)$ is trivial, since if a GO space is $[\nu, \nu]$-compact, then it has no (pseudogap of type $\nu$. Cf. the proof of $4.1(2) \Rightarrow(3)$.

Hence (1)-(4) are all equivalent.

$(3) \Rightarrow(5)$ is similar to $(3) \Rightarrow(4)$.

$(5) \Rightarrow\left(5^{\prime}\right)$ is trivial.

$\left(5^{\prime}\right) \Rightarrow(1)$, again, is trivial, since if a GO space is $[\nu, \nu]$-compact, then it satisfies all the conditions (2)-(4), (6)-(7) in 4.1, for that given $\nu$.

Hence (1)-(5) are all equivalent.

The equivalence of (3) and (6) is purely set-theoretical. (6) $\Rightarrow(3)$ is trivial since $H$ consists only of regular cardinals. For the converse, suppose that $F=$ $\left\{\nu_{1}, \ldots, \nu_{n}\right\}$, and let $D_{\nu_{1}}, \ldots, D_{\nu_{n}}$ be the corresponding ultrafilters given by (3). We are going to show that $D_{F}=D_{\nu_{1}} \times \cdots \times D_{\nu_{n}}$ works in (6). Recall that, for $D, D^{\prime}$ ultrafilters, say, over $I, I^{\prime}$, their product $D \times D^{\prime}$ is the ultrafilter over $I \times I^{\prime}$ defined by $Z \in D \times D^{\prime}$ if and only if $\left\{i \in I \mid\left\{i^{\prime} \in I^{\prime} \mid\left(i, i^{\prime}\right) \in Z\right\} \in D^{\prime}\right\} \in D$. It follows from the last statement in Lip10, Proposition 7.1] that, for every regular cardinal $\nu$, the following holds: $\nu \in K_{D \times D^{\prime}}$ if and only if either $\nu \in K_{D}$, or $\nu \in K_{D^{\prime}}$ (the result in Lip10, Proposition 7.1] is stated in terms of $(\nu, \nu)$-regularity, but, when $\nu$ is regular, this is equivalent to $\nu$-decomposability). In other words,

(*) $K_{D \times D^{\prime}}^{R e g}=K_{D}^{R e g} \cup K_{D^{\prime}}^{R e g}$, for every pair of ultrafilters $D$ and $D^{\prime}$.

In particular, if $D_{F}$ is defined as above, then $K_{D_{F}}^{R e g}=\bigcup_{\nu=\nu_{1}, \ldots, \nu_{n}} K_{D_{\nu}}^{R e g}$. By (3) we have that $K_{D_{\nu}}^{R e g} \subseteq H$, for $\nu=\nu_{1}, \ldots, \nu_{n}$, hence $K_{D_{F}}^{R e g} \subseteq H$. Moreover, $\nu \in K_{D_{\nu}}$, for $\nu=\nu_{1}, \ldots, \nu_{n}$, hence $F \subseteq K_{D_{F}}^{R e g}$. We have proved (3) $\Rightarrow(6)$.

The equivalence of (3) and (7), too, can be proved in a set-theoretical way, by a small variation on Lip10, Proposition 7.6 (a) $\Leftrightarrow(\mathrm{b})$ ], considering the case $\chi=\omega$ there, since every ultrafilter is $\omega$-complete. Notice that the proof of Lip10, Proposition 7.6] uses $(\nu, \nu)$-regularity even for $\nu$ singular, hence a proof along those lines needs the assumption that $H$ includes the cofinalities of the extremes of $F$. In fact, the assumption is necessary, as we shall show in Remark 6.3.

We shall give here a proof of $(3) \Rightarrow(7)$ with a stronger topological flavor, and which relies heavily on Cai99.

As above, $(7) \Rightarrow(3)$ is trivial. To get the converse, we shall prove (1) $\Rightarrow(7)$. First, we need a lemma, an easy extension of [Cai99, Corollary 1.8(iii)]. $\square_{\text {to be continued }}$ 
Lemma 6.2. Suppose that $\mathcal{T}$ is a class of topological spaces, and $\lambda, \mu$ are infinite cardinals. If every product of members of $\mathcal{T}$ is both [cf $\mu, \mathrm{cf} \mu]$-compact, and $[\nu, \nu]$ compact, for every regular $\nu$ with $\lambda \leq \nu \leq \mu$, then every product of members of $\mathcal{T}$ is $[\lambda, \mu]$-compact.

Proof. The particular case cf $\mu \geq \lambda$ is [Cai99, Corollary 1.8(iii)], and the lemma can be proved in a similar way. Otherwise, in case cf $\mu<\lambda$, we can apply Cai99, Corollary 1.8(iii)] for every regular $\mu^{\prime}<\mu$, getting that every product of members of $\mathcal{T}$ is $\left[\lambda, \mu^{\prime}\right]$-compact, for every regular $\mu^{\prime}<\mu$, and this, together with $[\operatorname{cf} \mu, \operatorname{cf} \mu]$ compactness immediately implies $[\lambda, \mu]$-compactness.

Proof of 6.1 (continued). We have promised a proof of $(1) \Rightarrow(7)$. So, let $F=$ $\operatorname{Reg} \cap \bigcup_{p \in P}\left[\lambda_{p}, \mu_{p}\right]$, with $P$ finite, and cf $\mu_{p} \in H$, for every $p \in P$. We have from (1) that every product of $H$-compact spaces is still $H$-compact. Applying Lemma 6.2 to the family $\mathcal{T}$ of all $H$-compact spaces, since $F$ is assumed to be a subset of $H$ and since $H$ includes the cofinalities of the extremes of $F$, we get that, for every $p \in P$, every product of $H$-compact spaces is $\left[\lambda_{p}, \mu_{p}\right]$-compact. By Cai99, Theorem 3.4], for every $p \in P$, there is a $\left(\lambda_{p}, \mu_{p}\right)$-regular ultrafilter $D_{p}$ such that every $H$-compact space is $D_{p}$-compact. As in the proof of $(2) \Rightarrow(3)$, for every regular cardinal $\kappa \notin H$, the space $\kappa$ with the order topology is $H$-compact, hence $D_{p}$-compact, and then, using again [Lip96. Proposition 1], we get that $D_{p}$ is not $\kappa$-decomposable, thus $\kappa \notin K_{D_{p}}^{R e g}$. Since this holds for every regular $\kappa \notin H$, then $K_{D_{p}}^{R e g} \subseteq H$. Enumerating $P$ as $\left\{p_{1}, \ldots, p_{n}\right\}$, and putting $D_{F}=D_{p_{1}} \times \cdots \times D_{p_{n}}$, we get $K_{D_{F}}^{R e g} \subseteq H$, by using $\left(^{*}\right)$ above. Moreover, a $(\lambda, \mu)$-regular ultrafilter is $\nu$ decomposable, for every regular $\nu$ such that $\lambda \leq \nu \leq \mu$ (see, e. g., Lip10, Property 1.1(xii)]), thus $\left[\lambda_{p}, \mu_{p}\right] \cap \operatorname{Reg} \subseteq K_{D_{p}}$, for every $p \in P$. Applying again (*), we get that $F \subseteq K_{D_{F}}$. In conclusion, $D_{F}$ witnesses (7).

To complete the proof of Corollary 6.1 it remains to prove $(7) \Leftrightarrow(8)$ under the additional assumption, but this is trivial, by taking $F=H$ (of course, in case $H$ is finite, it is easier to use (6)).

Remark 6.3. In general, the additional assumption before condition (8) in Corollary 6.1 is necessary in order to prove the equivalence of (8) with the other conditions.

First, we are going to show that (3) does not necessarily imply (8), when $H$ is infinite. Suppose that there are infinitely many measurable cardinals $\mu_{1}<\mu_{2}<\ldots$, and let $H=\left\{\mu_{n} \mid n \in \omega\right\}$. If $\mu$ is a measurable cardinal, there is a $\mu$-complete uniform ultrafilter $D_{\mu}$ over $\mu$, thus $K_{D_{\mu}}=\mu=K_{D_{\mu}}^{R e g}$. This implies that condition (3) in 6.1 holds for the above $H$.

However, if we further assume GCH, there is no ultrafilter $D$ such that $K_{D}^{R e g}=$ $H$. Indeed, the last paragraph in [Lip10, Section 7] shows that if $D$ is such an ultrafilter, then $D$ is $(\kappa, \kappa)$-regular, for $\kappa=\sup _{n \in \omega} \mu_{n}$. But then Lip10, Corollary $5.8]$ implies that $D$ is either cf $\kappa$-decomposable, or $\kappa^{+}$-decomposable, thus $K_{D}^{R e g}$ strictly contains $H$, a contradiction (notice that $\mathrm{cf} \kappa=\omega$ ).

There is also a similar counterexample in which $H$ is an interval, but $H$ does not include the cofinality of its upper extreme. Suppose that $\kappa$ is $\kappa^{+n}$-compact, for every $n \in \omega$. Here $\kappa^{+n}$ is $\kappa^{+\cdots+}$ with $n$ occurrences of "+". Let $\kappa^{+\omega}=$ $\sup _{n \in \omega} \kappa^{+n}$ and $H=\left[\kappa, \kappa^{+\omega}\right)=\operatorname{Reg} \cap\left[\kappa, \kappa^{+\omega}\right]$. By $\kappa^{+n}$-compactness, there is some $\kappa$-complete $\left(\kappa, \kappa^{+n}\right)$-regular ultrafilter $D_{n}$, thus $K_{D_{n}}=K_{D_{n}}^{R e g}=\left[\kappa, \kappa^{+n}\right]$. Hence $H$ satisfies condition (3) in 6.1 However, there is no ultrafilter $D$ such that 
$K_{D}^{R e g}=H$. Indeed, by Lip10, Theorem 5.7 and Corollary 5.8], such a $D$ would be either $\kappa^{+\omega+1}$-decomposable, or of $\kappa^{+\omega}$-decomposable, that is, $\omega$-decomposable; but both the above assertions contradict $K_{D}^{R e g}=H$. Thus (8) fails.

Notice that this last counterexample also shows that the assumption that $H$ includes the cofinalities of the extremes of $F$ is necessary in (7): just take $F=H$ to make (7) fail.

Corollary 6.1 shows that certain properties of products of GO spaces depend heavily on the set theoretical universe in which we work. If there is no inner model with a measurable cardinal, then, for every ultrafilter $D, K_{D}$ is always an interval of cardinals with minimum $\omega$, by results from Donder Don88, which extends and generalizes former results by M. Benda, C. C. Chang, R. B. Jensen, J. Ketonen, K. Prikry, J. H. Silver, among many others. See [Lip10, p. 363] for details. On the other hand, modulo some large cardinal consistency assumptions, it is possible to have an ultrafilter $D$ for which $K_{D}^{R e g}=\left\{\omega, \omega_{\omega+1}\right\}$, Ben-David and Magidor BDM86, Apter and Henle AH92. This is just an example concerning relatively small cardinals; many results are known, and nevertheless deep problems are still open about the possible values that the set $K_{D}$ can assume. See Problem 6.8 in Lip10, and the comments below it. By Corollary 6.1 (e. g., (1) $\Leftrightarrow(3)$ ), all these problems affect the behavior of GO spaces with respect to products, thus the use of ultrafilters in the results of the present section proves to be irreplaceable. We shall explicitly state just the example dealing with the smallest possible cardinals.

Corollary 6.4. (Assuming the consistency of a strongly compact cardinal) If $H=$ $\left\{\omega, \omega_{\omega+1}\right\}$, then the preservation of $H$-compactness under products in the class of GO spaces is both relatively consistent and independent from the axioms of ZFC.

Proof. Immediate from Corollary $6.1(1) \Leftrightarrow(8)$, and the just mentioned results by Donder, Ben-David and Magidor, and Apter and Henle.

Remark 6.5. Notice that $H=\left\{\omega, \omega_{\omega+1}\right\}$ is the "lowest" set for which independence can occur. Indeed, Cai99, Corollary 1.8(ii)] proves that if every product of members of a family $\mathcal{T}$ is $\left[\lambda^{+}, \lambda^{+}\right]$-compact, then every product of members of $\mathcal{T}$ is $[\lambda, \lambda]$ compact. Iterating, we get that if all products of members of $\mathcal{T}$ (whether $\mathcal{T}$ consists of GO spaces or not) are $\left[\omega_{n}, \omega_{n}\right]$-compact, then all such products are $\left[\omega_{i}, \omega_{i}\right]$ compact, for all $i \leq n$, hence also initially $\omega_{n}$-compact. Moreover, if there is some infinite cardinal $\lambda$ such that every product of members of some family $\mathcal{T}$ is $[\lambda, \lambda]$ compact, then the smallest such $\lambda$ is either $\omega$, or a measurable cardinal (hence it cannot be $\omega_{\omega+1}$ ). This is a slight generalization (with essentially the same proof) of [Cai99, Corollary 1.8(i)].

\section{Relationships Between $H$-COMPACTNESS AND OMISSION OF GAPS IN $H$}

The results in the present paper suggest that it is interesting to study the following property, depending on a class $H$ of infinite regular cardinals.

$\left({ }^{* *}\right)_{H}$ For every GO space $X, X$ is $[H]$-compact if and only if $X$ has no (pseudogap of type in $H$.

In general, $\left({ }^{* *}\right)_{H}$ is false; for example, take $H=\{\lambda\}$ with $\omega<\lambda \leq \mathfrak{c}$; then $\mathbb{R} \times \mathbb{Z}$ with the lexicographic order furnishes a counterexample to $(* *)_{H}$.

However, $\left({ }^{* *}\right)_{H}$ holds in many interesting cases. The simplest affirmative case is given already by Gulden, Fleischman and Weston result in the case of LOTS, or 
Corollary $5.1(2) \Leftrightarrow(4)$ here for general GO spaces. In this sense, the corollary states that $\left({ }^{* *}\right)_{H}$ holds in case $H=\operatorname{Reg} \cap[\omega, \lambda]$. Another example is given by Corollary 6.1, which implies that $\left({ }^{* *}\right)_{H}$ holds in case $H=K_{D}^{R e g}$, for some ultrafilter $D$.

Notice that, for $\lambda$ regular, if a GO space is $[\lambda, \lambda]$-compact, then, trivially, it has no (pseudo-)gap of type $\lambda$; hence, in order to prove an instance of $\left({ }^{* *}\right)_{H}$, it is enough to prove the non trivial implication. In order to give some results about $\left({ }^{* *}\right)_{H}$ we need some further definitions.

Suppose that $\lambda$ is an infinite cardinal, $X$ is a linearly ordered set, and $Y \subseteq X$. If $Y$ has no maximum, we say that $Y$ is $\lambda$-full (in $X$ ) on the right if, for every $y \in Y$, $\bigcup_{y^{\prime} \in Y}\left(y, y^{\prime}\right)_{X}$ has cardinality $\geq \lambda$. The subscript $X$ in $\left(y, y^{\prime}\right)_{X}$ is intended to mean that the interval is evaluated in $X$, that is, $\left(y, y^{\prime}\right)_{X}=\left\{x \in X \mid y<x<y^{\prime}\right\}$. When $X$ is clear from the context, we shall omit it. Symmetrically, if $Y$ has no minimum, $Y$ is $\lambda$-full (in $X$ ) on the left if, for every $y \in Y, \bigcup_{y^{\prime} \in Y}\left(y^{\prime}, y\right)_{X}$ has cardinality $\geq \lambda$. For sake of brevity, if $\alpha$ is an ordinal, we say that a linear order $Y$ is $\alpha \pm$ ordered in

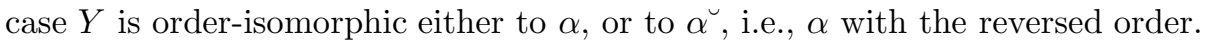

The next lemma shows that $\lambda$-full subsets always exist, for linear orders of cardinality $\geq \lambda$.

Lemma 7.1. Suppose that $\lambda$ is an infinite regular cardinal. Then every linearly ordered set $X$ of cardinality $\lambda$ either has a $\lambda \pm$ ordered subset, or a $\lambda$-full subset of order type $\omega$.

Proof. Suppose that there is $Y \subseteq X$ such that $|Y|=\lambda$ and, for every $y \in Y$, either $(-\infty, y)_{Y}$ or $(y, \infty)_{Y}$ has cardinality $<\lambda$. Then an easy induction of length $\lambda$, using the regularity of $\lambda$, shows that $Y$ (and hence $X$ ) has a $\lambda \pm$ ordered subset. Otherwise, for every $Y \subseteq X$ of cardinality $\lambda$, there is $y \in Y$ such that both $(-\infty, y)_{Y}$ and $(y, \infty)_{Y}$ have cardinality $\lambda$. Then an induction of length $\omega$ produces a strictly increasing sequence $\left(y_{n}\right)_{n \in \omega}$ such that $\left\{y_{n} \mid n \in \omega\right\}$ is $\lambda$-full in $X$.

Proposition 7.2. Suppose that $\nu \leq \lambda$ are regular cardinals. Then the following conditions are equivalent.

(1) Every linearly ordered set of cardinality $\lambda$ has either a $\lambda \pm$ ordered subset, or a $\nu \pm$ ordered $\lambda$-full subset.

(2) For every GO space $X$, if $X$ has no (pseudo-)gap of type $\nu$ or $\lambda$, then $X$ is $[\lambda, \lambda]$-compact.

(3) For every LOTS $X$, if $X$ has no gap of type $\nu$ or $\lambda$, then $X$ is $[\lambda, \lambda]$-compact.

In Conditions (2) and (3) we can equivalently restrict ourselves to those $X$ 's such that $|X| \leq \lambda^{\nu}$.

Proof. (Sketch) Recall that if $\lambda$ is regular, then $[\lambda, \lambda]$-compactness is equivalent to $\mathrm{CAP}_{\lambda}$. We shall use this equivalent condition throughout the proof.

(1) $\Rightarrow(2)$ Let $X$ be a GO space, $Z \subseteq X$, and $|Z|=\lambda$. Apply (1) to $Z$, say, $Z$ has subset $Y$ of order type $\nu$ and $\lambda$-full in $Z$. Since $X$ has no gap of type $\nu$, there is in $X$ a supremum $y$ of $Y$, and $y$ belongs to the closure of $Y$. Then every neighborhood of $y$ contains $\lambda$-many elements from $Z$, since $Y$ is $\lambda$-full in $Z$; in particular, $\lambda$-many elements from $X$, since $X \supseteq Z$.

The other cases are treated in a similar way.

$(2) \Rightarrow(3)$ is trivial.

$(3) \Rightarrow(1)$ We shall sketch a proof of the contrapositive. So, suppose that (1) fails, thus there exists a linear order $L$ of cardinality $\lambda$ without $\lambda \pm$ ordered subsets 
and without $\nu \pm$ ordered $\lambda$-full subsets. By Lemma 7.1, necessarily $\nu>\omega$. Extend $L$ to another linearly ordered set $X$ in the following way. Fill each gap having both left and right type $\nu$ by adding an element in the middle of the gap. If the gap has left type $\nu$ but a different right type, "fill" the gap by adding a copy of $\omega$. In the symmetric situation, add a copy of $\omega$. This procedure destroys all gaps of type $\nu$. We also want to "forbid" the existence (in $X$ ) of complete accumulation points of $L$. A candidate for such an accumulation point is some $\ell \in L$ such that, say, $(\ell, \infty)_{L}$ is $\lambda$-full in $L$ on the left. Then replace $\ell$ with a copy of $\omega$ or, according to the situation, with a copy of $\mathbb{Z}$. In the symmetric situation, it might be necessary to replace $\ell$ with a copy of $\omega$, instead.

Formally, $X$ can be considered as a subset of $L^{+} \times \mathbb{Z}$ with the lexicographical order, where $L^{+}$is the completion of $L$. It can be checked that the above constructions introduce no new gap of type $\nu$ or $\lambda$ ( $L$ has no gap of type $\lambda$ from the beginning), and that no point of $X$ is a complete accumulation point of $L$ (here we need the assumption that L has no $\nu \pm$ ordered $\lambda$-full subset otherwise the above "fillings" of gaps could introduce some complete accumulation point). Since $|L|=\lambda$, then (3) fails.

In order to prove the last statement it is enough to check that the $X$ constructed in the last part of the proof has cardinality $\leq \lambda^{\nu}$.

Corollary 7.3. If $H$ is a set of regular cardinals, then the following holds.

(1) If $H=\{\lambda\}$ is a singleton, then $\left({ }^{* *}\right)_{H}$ holds if and only if $\lambda$ is either $\omega$, or a weakly compact cardinal.

(2) If $\left({ }^{* *}\right)_{H}$ holds, then $\inf H$ is either $\omega$ or a weakly compact cardinal.

(3) If $\inf H=\omega$, then $\left({ }^{* *}\right)_{H}$ holds.

Proof. (1) is the particular case $\nu=\lambda$ of Proposition $7.2(2) \Leftrightarrow(1)$, since if $\lambda$ is regular, then every $\lambda \pm$ ordered subset is necessarily $\lambda$-full. Recall that, by a wellknown characterization, an uncountable cardinal $\lambda$ is weakly compact if and only if every linear order of cardinality $\lambda$ has a $\lambda \pm$ ordered subset. Notice that $\omega$, too, satisfies the above property.

(2) Let $\lambda=\inf H$. If $\lambda=\omega$, we are done. Otherwise, suppose by contradiction that $\lambda$ is not weakly compact, thus there is linear order $L$ without a $\lambda \pm$ ordered subset. Consider the LOTS $X=L \times \mathbb{Z}$, with the lexicographic order. Then $X$ has no gap of type $\lambda$, since $\lambda>\omega$; moreover, it has no gap of type $\nu \in H \backslash\{\lambda\}$, since $|X|=\lambda=\inf H<\nu$. By $\left({ }^{* *}\right)_{H}, X$ is $[\lambda, \lambda]$-compact, a contradiction, since $X$ has cardinality $\lambda$ and the discrete topology.

(3) Suppose that a GO space $X$ has no (pseudo-)gap of type in $H$. By Lemma 7.1, for every $\lambda \in H$, condition (1) in Proposition 7.2 is satisfied, when $\nu=\omega$. By Proposition $7.2(1) \Rightarrow(2), X$ is $[\lambda, \lambda]$-compact. This happens for every $\lambda \in H$, hence $X$ is $[H]$-compact.

\section{DISCLAIMER}

Though the author has done his best efforts to compile the following list of references in the most accurate way, he acknowledges that the list might turn out to be incomplete or partially inaccurate, possibly for reasons not depending on him. It is not intended that each work in the list has given equally significant contributions to the discipline. Henceforth the author disagrees with the use of the list (even in aggregate forms in combination with similar lists) in order to determine rankings 
or other indicators of, e. g., journals, individuals or institutions. In particular, the author considers that it is highly inappropriate, and strongly discourages, the use (even in partial, preliminary or auxiliary forms) of indicators extracted from the list in decisions about individuals (especially, job opportunities, career progressions etc.), attributions of funds, and selections or evaluations of research projects.

\section{REFERENCES}

[AH92] Arthur W. Apter and James M. Henle, On box, weak box and strong compactness, The Bulletin of the London Mathematical Society 24 (1992), no. 6, 513-518.

[AU29] P. Alexandroff and P. Urysohn, Mémoire sur les espaces topologiques compacts dédié à Monsieur D. Egoroff., Verhandelingen Amsterdam 14, No.1, 93 S., 1929.

[BDM86] Shai Ben-David and Menachem Magidor, The weak $\square^{*}$ is really weaker than the full $\square$, The Journal of Symbolic Logic 51 (1986), no. 4, 1029-1033.

[Ber70] Allen R. Bernstein, A new kind of compactness for topological spaces, Polska Akademia Nauk. Fundamenta Mathematicae 66 (1969/1970), 185-193.

[BL02] Harold R. Bennett and David J. Lutzer, Recent developments in the topology of ordered spaces, Recent progress in general topology, II (Miroslav Hušek and Jan van Mill, eds.), North-Holland, Amsterdam, 2002, pp. 83-114.

[Cai99] Xavier Caicedo, The abstract compactness theorem revisited, Logic and foundations of mathematics (Florence, 1995) (Andrea Cantini, Ettore Casari, and Pierluigi Minari, eds.), Synthese Lib., vol. 280, Kluwer Acad. Publ., Dordrecht, 1999, Selected papers from the 10th International Congress of Logic, Methodology and Philosophy of Science held in Florence, August 1995, pp. 131-141.

[CK90] C. C. Chang and H. J. Keisler, Model theory, third ed., Studies in Logic and the Foundations of Mathematics, vol. 73, North-Holland Publishing Co., Amsterdam, 1990.

[Don88] Hans-Dieter Donder, Regularity of ultrafilters and the core model, Israel Journal of Mathematics 63 (1988), no. 3, 289-322.

[GF99] Salvador García-Ferreira, On two generalizations of pseudocompactness, Topology Proceedings 24 (1999), 149-172 (2001).

[GFS97] Salvador García-Ferreira and Manuel Sanchis, On C-compact subsets, Houston Journal of Mathematics 23 (1997), no. 1, 65-86.

[GFW70] Samuel L. Gulden, William Fleischman, and J. H. Weston, Linearly ordered topological spaces, Proceedings of the American Mathematical Society 24 (1970), 197-203.

[GS75] John Ginsburg and Victor Saks, Some applications of ultrafilters in topology, Pacific Journal of Mathematics 57 (1975), no. 2, 403-418.

[Kan86] Akihiro Kanamori, Finest partitions for ultrafilters, The Journal of Symbolic Logic 51 (1986), no. 2, 327-332.

[Lip96] Paolo Lipparini, Productive $[\lambda, \mu]$-compactness and regular ultrafilters, Topology Proceedings 21 (1996), 161-171.

[Lip10] - More on regular and decomposable ultrafilters in ZFC, MLQ. Mathematical Logic Quarterly 56 (2010), no. 4, 340-374.

[Lip11a] _ Every weakly initially $\mathfrak{m}$-compact topological space is $\mathfrak{m} P C A P$, Czechoslovak Mathematical Journal 61(136) (2011), no. 3, 781-784.

[Lip11b] More generalizations of pseudocompactness, Topology and its Applications 158 (2011), no. 13, 1655-1666.

[Lip12] Some compactness properties related to pseudocompactness and ultrafilter convergence, Topology Proceedings 40 (2012), 29-51.

[Lip13] - Initial $\lambda$-compactness in linearly ordered spaces, 2013, arXiv:1306.1715

[Lip14] Topological spaces compact with respect to a set of filters, Central European Journal of Mathematics 12 (2014), no. 7, 991-999.

[Nag85] Jun-iti Nagata, Modern general topology, second ed., North-Holland Mathematical Library, vol. 33, North-Holland Publishing Co., Amsterdam, 1985.

[NV87] P. J. Nyikos and J. E. Vaughan, Sequentially compact, Franklin-Rajagopalan spaces, Proceedings of the American Mathematical Society 101 (1987), no. 1, 149-155.

[Pur73] Steven D. Purisch, On the orderability of Stone-Čech compactifications, Proceedings of the American Mathematical Society 41 (1973), 55-56. 
[Ret93] Teklehaimanot Retta, Some cardinal generalizations of pseudocompactness, Czechoslovak Mathematical Journal 43(118) (1993), no. 3, 385-390.

[Sak78] Victor Saks, Ultrafilter invariants in topological spaces, Transactions of the American Mathematical Society 241 (1978), 79-97.

[Ste84] R. M. Stephenson, Jr., Initially $\kappa$-compact and related spaces, Handbook of set-theoretic topology (Kenneth Kunen and Jerry E. Vaughan, eds.), North-Holland, Amsterdam, 1984 , pp. 603-632.

[SV74] R. M. Stephenson, Jr. and J. E. Vaughan, Products of initially m-compact spaces, Transactions of the American Mathematical Society 196 (1974), 177-189.

[Vau84] Jerry E. Vaughan, Countably compact and sequentially compact spaces, Handbook of set-theoretic topology (Kenneth Kunen and Jerry E. Vaughan, eds.), North-Holland, Amsterdam, 1984, pp. 569-602.

Dipartimento di Matematica, Viale degli Ordini Scientifici, II Università di Roma (TOR VERGATA), I-00133 ROME ITALY

URL: http://www.mat.uniroma2.it/ 〜ipparin

E-mail address: lipparin@axp.mat.uniroma2.it 